\title{
Holder, Gilles. - Poussière, Ô poussière
}

Paris, Société d'ethnologie, 2001, 503 p.

\section{Anne Doquet}

\section{(2) OpenEdition}

\section{Journals}

Édition électronique

URL : http://journals.openedition.org/etudesafricaines/4792

DOI : 10.4000/etudesafricaines.4792

ISSN : $1777-5353$

Éditeur

Éditions de l'EHESS

Édition imprimée

Date de publication : 1 janvier 2004

Pagination : 698-700

ISBN : 978-2-7132-2004-3

ISSN : 0008-0055

\section{Référence électronique}

Anne Doquet, «Holder, Gilles. - Poussière, Ô poussière », Cahiers d'études africaines [En ligne], 175 |

2004, mis en ligne le 13 mars 2007, consulté le 24 septembre 2020. URL : http://

journals.openedition.org/etudesafricaines/4792; DOI : https://doi.org/10.4000/etudesafricaines.4792

Ce document a été généré automatiquement le 24 septembre 2020.

(C) Cahiers d'Études africaines 


\section{Holder, Gilles. - Poussière, Ô poussière}

Paris, Société d'ethnologie, 2001, 503 p.

\section{Anne Doquet}

1 En prenant comme objet d'étude la cité-État Sama du Pays Dogon, Gilles Holder introduit un concept jusqu'alors absent de l'anthropologie politique africaniste. C'est en effet comme une cité-État qu'est décrite la petite ville de Kani-Gogouna, située au cœur d'une des régions favorites de l'ethnologie française. L'ouvrage se présente sous forme de trois livres, relativement indépendants les uns des autres, qui illustrent respectivement la fondation, la constitution et l'évolution de cette cité-État.

2 Le premier livre est consacré aux questions de l'identité dans l'histoire et de l'histoire dans l'identité. L'identité y est lue comme un enjeu politique pour les Saman, qui se définissent dans une histoire guerrière. La précision et la rigueur de cette restitution de l'histoire permettent de comprendre la complexité identitaire des Saman, tels qu'ils se définissent eux-mêmes ou tels qu'ils sont qualifiés de l'extérieur. Dotés dans le temps comme dans l'espace d'ethnonymes variables dénotant le flou identitaire qui entoure ce peuple peu étudié, les Saman s'auto-définissent comme "ceux de Djenné ", et par conséquent comme héritiers de sa civilisation urbaine, ses pratiques musulmanes et son organisation politique. Avec un souci du détail rare, l'auteur déchiffre chaque étape de leur longue migration constituée de ruptures et de défaites, mais qui s'achève par une victoire et par leur installation sur le plateau dogon. Cette vision diachronique du temps est légitimée par l'importante place que lui concèdent les Saman : l'histoire est en effet un facteur de légitimation territoriale, dans la mesure où c'est par leur passé guerrier et par la fondation de leur cité qu'ils se définissent. Au temps linéaire de l'histoire se superpose par suite le mouvement cyclique du mythe. À la croisée de l'histoire et du mythe, mais aussi de l'islam et du paganisme, du «Livre » et du "Fétiche», les Saman font montre de leur sentiment de former un peuple culturellement singulier, ce qui amène l'auteur à parler de Nation.

3 Le deuxième livre décrit la fondation de cette cité, illustrant ainsi la réalisation de la nation. Partant de la morphologie de la localité, enceinte-frontière, Holder examine 
l'appréhension de l'espace de la cité par la périphérie et le centre et décrit le jeu d'alliance entre Saman et Dogon qui le légitiment. Au sein de cette anthropologie de l'espace sont éclairées les notions de citadinité et surtout de citoyenneté, qui sous tendent la question de l'esclavage. La question du territoire de la cité-État est alors envisagée sous l'angle de sa souveraineté politique, la cité se légitimant non par des droits sur la terre, mais par des droits sur des hommes, en particulier par l'usage de captifs publics. L'espace politique est ensuite suivi dans l'histoire, la période du Kalhifat des Fulbe de Hamdallaye ayant induit un glissement des institutions vers une plus grande autonomie politique.

4 Consacré à l'exercice de cette cité-État, le troisième livre est dominé par l'idée de Force chez les Saman, concept déterminant dans la constitution et le maintien de leur organisation politique. En tant que production logique de l'autorité, la Force, incarnée par un roi sacré, devait être proclamée sans être utilisée. Lorsque son usage fut imposé par l'histoire, la cité-État se disloqua au profit de nouvelles formes d'organisation politique, tel l'État-territoire. L'ouvrage s'achève enfin par l'interprétation des grands rites royaux, notamment celui du régicide, qui ramènent le lecteur aux différents concepts-clés précédemment traités : l'histoire, le mythe, l'identité, et la réalisation spatiale et temporelle de la cité-État, et constituent l'ultime témoignage de l'existence de cette nation sama.

On ne peut que féliciter Gilles Holder pour la rigueur et la précision de son écriture dans la restitution de l'histoire de cette cité-État un peu oubliée. On peut néanmoins regretter que la formation historique de l'auteur prenne le pas sur la perspective anthropologique de l'ouvrage et notamment que le travail ne fasse que peu de place aux représentations contemporaines de l'identité saman. Lorsque Holder écrit que les «principes sous-jacents de la cité-État demeurent» (p.36) ou que «l'idée de citoyenneté n'a pas encore totalement disparu » (p. 472), il laisse quelque peu le lecteur sur sa faim quant aux résidus identitaires de cette cité. De même, les extraits d'entretiens qui émaillent l'ouvrage sont peu circonstanciés, alors que la période d'enquête en déterminait sans doute l'orientation. On peut également regretter le manque d'entretiens sur la perception que les Dogon ont des Saman aujourd'hui, même si l'auteur, présent sur le terrain au cours d'une période particulièrement délicate, s'en justifie (p. 22).

6 On ne peut cependant que louer le travail d'analyse méticuleux de Gilles Holder, qui décortique les mécanismes socio-politiques originaux de cette localité, mécanismes à propos desquels les grilles de l'anthropologie classique ne sont pas opératoires. D'aucuns pourront juger abusive la qualification de cité-État et encore plus de nation pour la petite ville de Kani-Gogouna. L'argumentation est pourtant précise et l'on ne peut, quoi qu'il en soit, qu'être persuadé qu'à la typologie classique de l'anthropologie africaniste faisaient jusqu'à présent défaut les termes pour qualifier une telle configuration. Au-delà d'une connaissance approfondie des Saman «inconnus", Poussière, $\hat{O}$, poussière invite donc l'anthropologie politique, encore aujourd'hui entachée d'ethnocentrisme, à élargir la panoplie des structures politiques possibles des sociétés africaines. En outre, cet ouvrage a le mérite d'illustrer le caractère hétérogène et fluctuant de l'identité, en présentant une petite société dont les expressions identitaires, jonglant avec l'Histoire et le mythe, tout autant qu'avec le Livre et le Fétiche, allient des catégories traditionnellement réservées à des types de sociétés particuliers. 
7 Ainsi, le livre de Gilles Holder bat en brèche, par la rigueur de son argumentation, la conception unifiante et idéalisée du Pays Dogon héritée de l'école de Marcel Griaule, et en cela il participe au renouveau en cours de l'anthropologie des Dogon. Contribuant à éclairer les mouvements identitaires dans l'histoire d'une région toujours envisagée en dehors de l'histoire, il déconstruit l'appréhension anthropologique habituelle d'une culture souvent envisagée comme un isolat. 